\title{
Practical Considerations for High-Resolution Transmission Kikuchi Diffraction Mapping and Analysis in Titanium Alloys
}

Jonathan Orsborn ${ }^{1,2}$, Genevieve Lee ${ }^{3}$, S. A. Romo ${ }^{3}$, Thomas F. Broderick ${ }^{4}$, Antonio J. Ramirez ${ }^{3}$, David W. McComb ${ }^{2}$, Hamish L. Fraser ${ }^{1}$

1. Center for the Accelerated Maturation of Materials, Department of Materials Science and Engineering, The Ohio State University, Columbus, $\mathrm{OH}$

2. Center for Electron Microscopy and Analysis, Department of Materials Science and Engineering, The Ohio State University, Columbus, $\mathrm{OH}$

3. Welding Engineering Program, Department of Materials Science and Engineering, The Ohio State University, Columbus, $\mathrm{OH}$

${ }^{4}$ G.E. Aviation, Materials Process Engineering Department, One Neumann Way, Cincinnati, OH

Transmission Kikuchi Diffraction (TKD), sometimes referred to as t-EBSD, is a very useful SEM-based technique for the rapid crystallographic orientation mapping and analysis of fine-grained materials at reasonably high spatial resolution[1, 2]. TKD is analogous to Electron Backscatter Diffraction (EBSD), but uses a thin foil (TEM specimen) instead of a bulk SEM specimen. The reduced interaction volume of the thin foil enables achievable resolutions of $10 \mathrm{~nm}$ or better[3-5]. The two techniques share so many similarities that TKD is actually quite easy for an experienced EBSD-user, with the only requirement beyond the traditional EBSD setup being an appropriate sample holder. While work has been done to investigate the effect of the geometry of the microscope environment, sample thickness, and beam conditions [1, 2], the ideal parameters depend on needs of the investigator. This study will touch on the effect of these parameters, while focusing more on often-overlooked factors such as sample preparation technique, stage/beam stabilization, carbon contamination, and sample charging, particularly as they relate to collecting high-resolution TKD maps in titanium alloys.

All of the work in this study was completed on an FEI XL-30 ESEM ${ }^{\mathrm{TM}}$, using a custom sample holder, and an EDAX Hikari ${ }^{\mathrm{TM}}$ camera. A wide variety of microscope environment geometries may be used; specimen tilt can vary between $0^{\circ}$ and $-60^{\circ}$, and working distance can vary from 3 to $15 \mathrm{~mm}$. However, it has been shown that shorter working distances result in more interpretable diffraction patterns on the EBSD camera[2]. The data for this work were all collected at working distances of either 4 or $5 \mathrm{~mm}$. It is also known that more sample tilt increases the effective foil thickness, resulting in more signal and better patterns on the camera[2]. While a greater sample tilt does produce better patterns, the greater effective foil thickness creates more beam-broadening, thus degrading the spatial resolution of the scan. Another issue that arises from increased effective foil thickness is that the electron dosage rate also increases, resulting in an increased likelihood of sample charging occurring during a scan. It has been asserted that lower accelerating voltages $(20$ or $25 \mathrm{kV}$, instead of $30 \mathrm{kV}$ ) may be useful for very thin specimens and lighter elements such as titanium[2]. However, this study found $30 \mathrm{kV}$ to produce better patterns and higher-resolution scans.

It has been established that the diffraction pattern on the camera is the result of diffraction that occurs in the bottom portion of the foil, i.e., from the exit surface[6]. Since the diffraction information comes from only a portion of the foil thickness, it is extremely important that the damage layer on the foil be as thin as possible. In this investigation, it was found that ion-milled bulk TEM specimens ( $3 \mathrm{~mm}$ disc) had damage layers that were too thick, in addition to too much variation in sample thickness to produce 
decent TKD maps. While camera parameters and image processing can compensate for slight variations in sample thickness, large variations in sample thickness interfere with image processing techniques. A very good sample preparation process was found to be FIB-milled samples, produced with initial ion energy of $30 \mathrm{kV}$, followed by $5 \mathrm{kV}$ ion beam cleaning. The pattern quality and map resolution were improved even further when the foil was subjected to $900 \mathrm{~V}$ and $500 \mathrm{~V}$ cleaning steps, in a Fischione Nanomill ${ }^{\mathrm{TM}}$. An example TKD map of $\alpha+\beta$ processed T-17, is displayed in Figure 1. These maps combine IPF and IQ maps, and display lattice icons representing crystallographic orientations. The sample was carefully prepared using an FEI Helios Focused Ion Beam, and cleaned in the Nanomill ${ }^{\mathrm{TM}}$, as previously described. The data were collected at a working distance of $4 \mathrm{~mm}$, a specimen tilt of $-20^{\circ}$, accelerating voltage of $30 \mathrm{kV}$, a $100 \mu \mathrm{m}$ aperture, camera exposure of $15 \mathrm{~ms}$, and $10 \mathrm{~nm}$ step size. The indexing was done automatically in the software via Hough transform peak indexing. Figure 1 shows the alpha-titanium and beta-titanium IPF maps overlaid with gray scale confidence index maps, displayed as the left and right images, respectively. Samples will also be investigated via TEM, to confirm the accuracy of the TKD.

\section{References:}

[1] Trimby, P.W., Orientation mapping of nanostructured materials using transmission Kikuchi diffraction in the scanning electron microscope. Ultramicroscopy, 2012. 120: p. 16-24.

[2] Suzuki, S., Features of Transmission EBSD and its Application. Jom, 2013. 65(9): p. 1254-1263.

[3] Keller, R.R. and R.H. Geiss, Transmission EBSD from $10 \mathrm{~nm}$ domains in a scanning electron microscope. Journal of Microscopy, 2012. 245(3): p. 245-251.

[4] Abbasi, M., et al., Application of Transmitted Kikuchi Diffraction in Studying Nano-oxide and Ultrafine Metallic Grains. ACS Nano, 2015. 9(11): p. 10991-11002.

[5] Brodusch, N., H. Demers, and R. Gauvin, Dark-field imaging of thin specimens with a forescatter electron detector at low accelerating voltage. Microsc Microanal, 2013. 19(6): p. 1688-97.

[6] Rice, K.P., R.R. Keller, and M.P. Stoykovich, Specimen-thickness effects on transmission Kikuchi patterns in the scanning electron microscope. J Microsc, 2014. 254(3): p. 129-36.
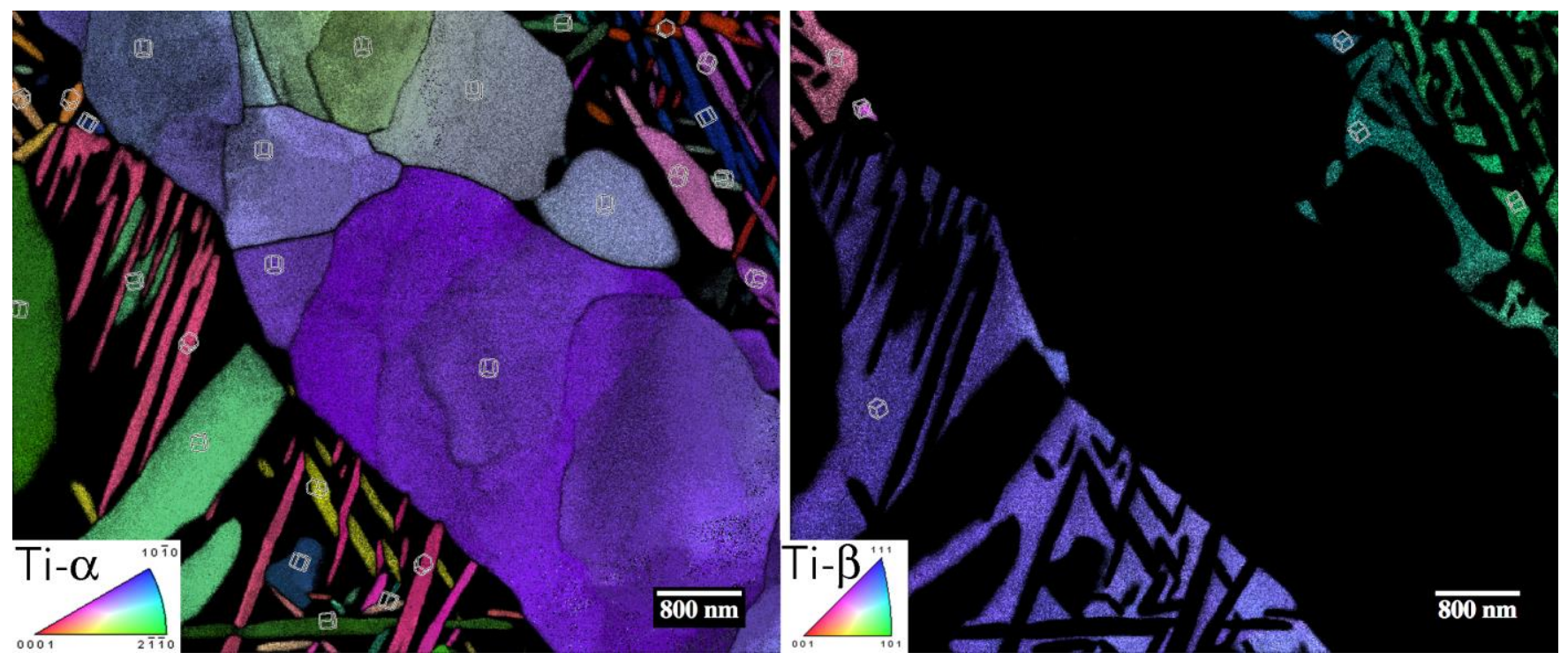

Figure 1: IPF+IQ maps for the alpha (left) and beta (right). Unique crystallographic orientations are highlighted with unit cell icons. 